\title{
Equilibration dynamics and conductivity of warm dense hydrogen
}

U. Zastrau, ${ }^{1,2, *}$ P. Sperling, ${ }^{3}$ A. Becker, ${ }^{3}$ T. Bornath,${ }^{3}$ R. Bredow, ${ }^{3}$ T. Döppner, ${ }^{4}$ S. Dziarzhytski, ${ }^{5}$ T. Fennel, ${ }^{3}$ L. B. Fletcher, ${ }^{2}$ E. Förster, ${ }^{1,6}$ C. Fortmann, ${ }^{4}$ S. H. Glenzer, ${ }^{2}$ S. Göde, ${ }^{2,3}$ G. Gregori, ${ }^{7}$ M. Harmand,${ }^{5}$ V. Hilbert, ${ }^{1}$ B. Holst, ${ }^{3}$ T. Laarmann, ${ }^{5,8}$ H. J. Lee, ${ }^{2}$ T. Ma, ${ }^{4}$ J. P. Mithen, ${ }^{7}$ R. Mitzner, ${ }^{9}$ C. D. Murphy,${ }^{7}$ M. Nakatsutsumi, ${ }^{10}$ P. Neumayer, ${ }^{11}$ A. Przystawik, ${ }^{5}$ S. Roling, ${ }^{9}$ M. Schulz, ${ }^{5}$ B. Siemer, ${ }^{9}$ S. Skruszewicz, ${ }^{3}$ J. Tiggesbäumker, ${ }^{3}$ S. Toleikis,${ }^{5}$ T. Tschentscher, ${ }^{10}$ T. White, ${ }^{7}$ M. Wöstmann, ${ }^{9}$ H. Zacharias, ${ }^{9}$ and R. Redmer ${ }^{3}$

${ }^{1}$ Institut für Optik und Quantenelektronik, Friedrich-Schiller-Universität, Max-Wien-Platz 1, 07743 Jena, Germany ${ }^{2}$ SLAC National Accelerator Laboratory, 2575 Sand Hill Road, Menlo Park, California 94025, USA

${ }^{3}$ Institut für Physik, Universität Rostock, D-18051 Rostock, Germany

${ }^{4}$ Lawrence Livermore National Laboratory, 7000 East Avenue, Livermore, California 94550, USA

${ }^{5}$ Deutsches Elektronen-Synchrotron, Notkestrasse 85, D-22607 Hamburg, Germany

${ }^{6}$ Helmholtz-Institut Jena, Fröbelstieg 3, 07743 Jena, Germany

${ }^{7}$ Department of Physics, Clarendon Laboratory, University of Oxford, Parks Road, Oxford OX1 3PU, United Kingdom

${ }^{8}$ The Hamburg Centre for Ultrafast Imaging, 22761 Hamburg, Germany

${ }^{9}$ Physikalisches Institut, Westfälische Wilhelms-Universität, Wilhelm-Klemm-Straße 10, 48149 Münster, Germany

${ }^{10}$ European XFEL, Albert-Einstein-Ring 19, 22761 Hamburg, Germany

${ }^{11}$ Extreme Matter Institute, GSI Helmholtzzentrum für Schwerionenforschung, 64291 Darmstadt, Germany

(Received 14 February 2014; published 18 July 2014)

\begin{abstract}
We investigate subpicosecond dynamics of warm dense hydrogen at the XUV free-electron laser facility (FLASH) at DESY (Hamburg). Ultrafast impulsive electron heating is initiated by a $\leqslant 300$-fs short $\mathrm{x}$-ray burst of 92-eV photon energy. A second pulse probes the sample via $\mathrm{x}$-ray scattering at jitter-free variable time delay. We show that the initial molecular structure dissociates within $(0.9 \pm 0.2) \mathrm{ps}$, allowing us to infer the energy transfer rate between electrons and ions. We evaluate Saha and Thomas-Fermi ionization models in radiation hydrodynamics simulations, predicting plasma parameters that are subsequently used to calculate the static structure factor. A conductivity model for partially ionized plasma is validated by two-temperature density-functional theory coupled to molecular dynamic simulations and agrees with the experimental data. Our results provide important insights and the needed experimental data on transport properties of dense plasmas.
\end{abstract}

DOI: 10.1103/PhysRevE.90.013104

PACS number(s): 52.20.Fs, 52.25.Os, 52.27.Gr, 52.50.Jm

\section{INTRODUCTION}

The thermodynamic properties of even the simplest element hydrogen remain elusive when it comes to extreme conditions of density, pressure, and temperature. Although not appearing naturally on earth, hydrogen is found in a diversity of extreme conditions in the solar system and beyond. Knowledge of the equation of state over a broad parameter range is hence critical for modeling stellar and planetary interiors [1], as well as for inertial confinement fusion (ICF) experiments [2].

The mechanisms causing deviation from the ideal gas law have to be taken into account in regions of phase transitions and partial ionization due to electron degeneracy and high density. Microscopic properties of matter related to reflectivity and electrical and thermal conductivity are tied to dynamic energy transport between electrons and ions. On a macroscopic scale, these affect the depth of mixing layers in Jovian planets [3], as well as the formation of a central hot spot and the assembly of a stable thermonuclear fuel layer in ICF implosions [4-6]. Experiments aiming at measuring these quantities hence probe the response of the system to an impulsive pump with high temporal resolution.

Presently, uncertainties in the transport properties in dense matter limit our ability to accurately model such complex systems. Recently, we reported on x-ray measurements in

*ulf.zastrau@uni-jena.de cryogenic hydrogen [7] where ultrafast electron heating is initiated by a short $x$-ray burst of duration $\leqslant 300$ fs. A second pulse probes the sample via $\mathrm{x}$-ray scattering at variable time delay. It was shown that the initial molecular structure dissociates within $0.9 \pm 0.2 \mathrm{ps}$.

This work discusses deeper aspects of the experiment that show that a proper description of the equilibration dynamics is far from being trivial. First, we show the impact of using different equation of states in radiation-hydrodynamics simulations and that our experiment provides a reference point against which ionization models for warm and dense matter states are validated. Further, we calculate the structure factor for x-ray scattering with single- and two-temperature approaches, based on the plasma temperatures from the hydrodynamics simulations, and validate the results against our experimental results. Finally, a conductivity model for partially ionized plasma is validated by experimental data and two-temperature density-functional theory coupled to molecular dynamic simulations.

\section{PUMP-PROBE SETUP}

As a versatile diagnostic tool for warm and dense matter states, x-ray scattering was demonstrated on picosecond [8] and nanosecond time scales [9-11]. With the advent of free-electron lasers (FELs), the implementation of volumetric $\mathrm{x}$-ray heating $[12,13]$ and accurate $\mathrm{x}$-ray scattering on the femtosecond time scale is now becoming possible due to the 


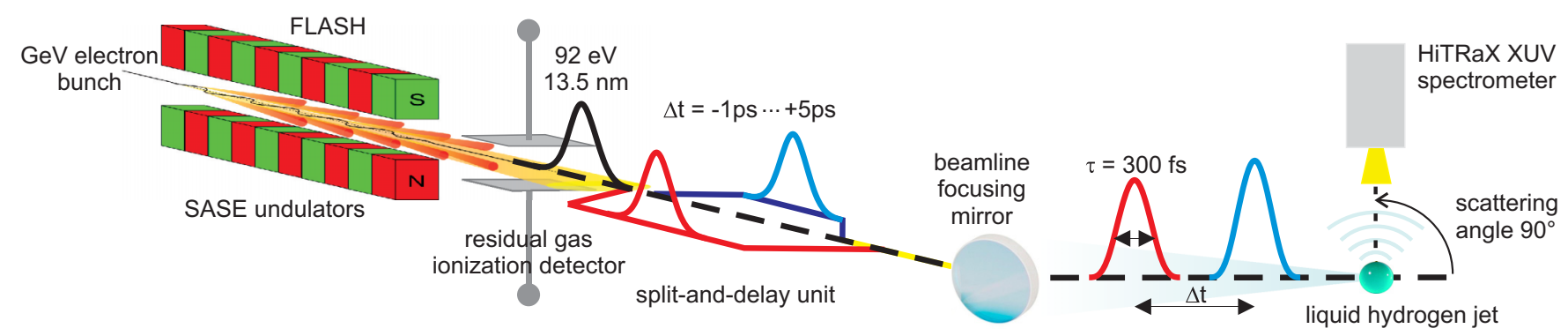

FIG. 1. (Color online) Schematic of the experimental setup (figure from Ref. [7]). Soft-X-ray FEL pulses are split and delayed before they are focused onto a cryogenic hydrogen jet. Scattering at $90^{\circ}$ is observed with an XUV spectrometer. For further details refer to the text.

short FEL pulse lengths ( $\leqslant 300 \mathrm{fs})$, their unprecedented peak brightness, and the high repetition rate [14].

To investigate dynamic processes of warm and dense matter on such short time scales, pump-probe experiments are necessary, where a first pulse generates an excited state that is subsequently probed by a second pulse at well-defined time delays. In a pioneering experiment [7] we used the unique split-and-delay capability of the free-electron laser in Hamburg (FLASH) [15] (as illustrated in Fig. 1) in order to volumetrically heat liquid hydrogen and subsequently probe it by ultrafast x-ray scattering.

The FLASH has been operated at $E=92 \mathrm{eV}$ photon energy with a spectral bandwidth of $\Delta E / E \approx 1.6 \%$. An upper limit for the FEL pulse duration of $\leqslant 300$ fs is estimated from the measured electron bunch duration ( 300-fs full width at half maximum). The bunch charge was $0.33 \mathrm{nC}$ and the bunch energy was $\sim 140 \mu \mathrm{J}$ at a repetition rate of $10 \mathrm{~Hz}$. The individual $x$-ray pulse energies have been recorded by a residual gas ionization detector [16], yielding an average initial pulse energy of $200 \pm 50 \mu \mathrm{J}$.

The horizontally polarized FEL radiation is geometrically divided by a split-and-delay unit (SDU) [15,17]. Likewise, a time delay between $\mathrm{x}$-ray pump and probe pulses is introduced with a precision of a few femtoseconds. The intensity ratio of the divided pulses is given by the relative split areas $A_{v}$ and $A_{f}$ for the variable and the fixed branch, respectively, multiplied by the transmission of the respective branch. Figure 2 shows a photograph of the exit port of the SDU. The pulses are

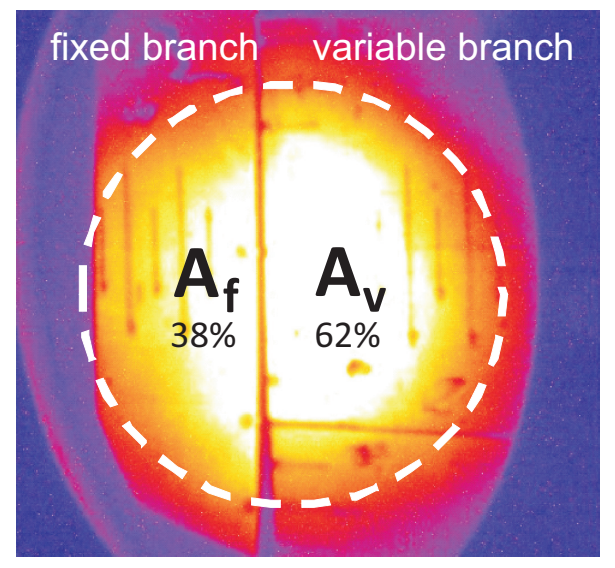

FIG. 2. (Color online) Relative geometrical areas $A_{f}$ and $A_{v}$ of the split-and-delay unit. subsequently focused to a $(20 \times 30)-\mu \mathrm{m}^{2}$ spot using the ellipsoidal carbon-coated beamline mirror, yielding intensities up to $27 \pm 0.6 \mathrm{TW} / \mathrm{cm}^{2}$ for the variable delay branch and $19 \pm 0.4 \mathrm{TW} / \mathrm{cm}^{2}$ for the fixed branch. See Table I for a detailed table showing the transmission of the beamline components.

In the focus, the FEL pulses hit a $(9 \pm 2)$ - $\mu$ m-radius cryogenic hydrogen jet with mass density of $0.08 \mathrm{~g} / \mathrm{cm}^{3}$ $\left(5 \times 10^{22} \mathrm{~cm}^{-3}\right)$ and temperature of $20 \mathrm{~K}$, prepared in a liquidhelium-cooled cryostat [18]. With a $60-\mathrm{m} / \mathrm{s}$ flow velocity each FLASH pulse scatters from an unperturbed sample. During hydrogen injection the vacuum chamber was at a pressure of $10^{-5}$ mbar.

\section{X-RAY HEATING}

For the pump pulse we have chosen 92-eV soft-x-ray radiation to be in a regime where the photon energy is well above the plasma frequency for liquid-density hydrogen. This

TABLE I. FLASH beamline efficiency for a 13.5-nm wavelength, accounting for the split-and-delay unit. The gas monitor detector is denoted by GMD and the variable line spacing beamline spectrometer by VLS. The beam line 2 at FLASH is denoted by BL2.

\begin{tabular}{lcc}
\hline \hline \multicolumn{2}{c}{ Beamline properties } \\
\hline GMD & & $(200 \pm 50) \mu \mathrm{J}$ \\
BL2 [16] & & $(64 \pm 4) \mu \mathrm{J}$ \\
VLS [16] & $\sin 0.9$ \\
Pulse duration & & $\leqslant 300 \mathrm{fs}$ \\
Focal spot area & Split and delay & \\
& Fixed-delay branch & \\
\hline & & \\
Relative split area $A_{f}$ & & 0.38 \\
Reflectivity [15,17] & & 0.75 \\
Total transmission & & 0.164 \\
Pulse energy & & $33.5 \mu \mathrm{J}$ \\
Power & & $1.12 \times 10^{8} \mathrm{~W}$ \\
Intensity & Variable-delay branch & \\
& & $0.4) \mathrm{TW} / \mathrm{cm}^{2}$ \\
Relative split area $A_{v}$ & & 0.62 \\
Reflectivity [15,17] & & 0.67 \\
Total transmission & & 0.239 \\
Pulse energy & & $48.8 \mu \mathrm{J}$ \\
Power & & $1.63 \times 10^{8} \mathrm{~W}$ \\
Intensity & & $(27 \pm 0.6) \mathrm{TW} / \mathrm{cm}^{2}$ \\
\hline \hline
\end{tabular}


leads to a high penetration depth with an absorption length $l_{\mathrm{abs}}=11 \mu \mathrm{m}$ [19], comparable to the hydrogen jet radius of $\sim 9 \mu \mathrm{m}$. The initial energy deposition via photoabsorption is not uniform, as FEL intensities of $I_{0}$ are present at the target front side, compared to only $\sim 0.2 I_{0}$ at the target rear side; however, the generated photoelectrons with kinetic energies of $78 \mathrm{eV}$ are capable of impact ionizing several molecules and atoms within the FEL pulse duration [20]. This leads to further ionization, molecular heating, expansion, and breaking of the covalent $\mathrm{H}-\mathrm{H}$ bond (dissociation). All these effects lead to an almost homogeneous electron heating throughout the sample.

\section{A. Radiation-hydrodynamics simulations}

To describe the temporal evolution of the plasma, one-dimensional radiation-hydrodynamics simulations are performed using the code HELIOS [21]. HELIOS solves the equations of motion for a fluid in the Lagrangian frame considering the electronic and ionic subsystem as comoving, while their energies are handled separately. The radiation emission and absorption are coupled to the electronic system, where the multifrequency radiation intensities are computed based on a radiation diffusion model. The equation of state (EOS) is provided by the PROPACEOS data package. The code uses nonlocal thermodynamic equilibrium atomic level populations on which the frequency-dependent opacities are based. The molecular character of the considered system is not taken into account by the HELIOS code.

Given that the electron-ion equilibration time is an unknown in virtually all high-density plasma experiments, we compare the predictions by HELIOS using two different EOS models. PROPACEOS 4.2 calculates the ionization via a Saha model, while PROPACEOS 5.1 uses a Thomas-Fermi model as implemented in the quotidian EOS (QEOS) [22]. In the remainder of this paper, we will refer to the first model as the Saha-like, while the latter will be referred to as QEOS-like.

The hydrodynamics simulations yield the spatially resolved ion density and the electron and heavy-particle temperatures. They were performed for each delay time using two 300-fs XUV pulses (pumping at 0 ps and probing after time), so the pulse duration is accounted for. The plasma conditions are extracted from these simulations at the peak of the probe pulse. In Fig. 3 the average values of the target are shown because temperature gradients are not pronounced. Spatial homogeneity had to be assumed because the subsequent calculations (see Sec. VA) would exceed the up-to-date computing capability for inhomogeneous targets.

\section{B. Ionization}

Saha's set of equations yields the ionization degree for a gas at equilibrium temperature $T$. The single equation for hydrogen gas has an analytic solution. Assuming most of the neutral hydrogen being in the ground state [the statistical weights being $g(\mathrm{H})=2$ and $g\left(\mathrm{H}^{+}\right)=1$ ] and charge is conserved, we may write

$$
\frac{Z_{\text {free }}^{2}}{1-Z_{\text {free }}}=\frac{1}{n}\left(\frac{2 \pi m_{e} k_{B} T}{h^{2}}\right)^{3 / 2} e^{-13.6 \mathrm{eV} / k_{B} T} .
$$

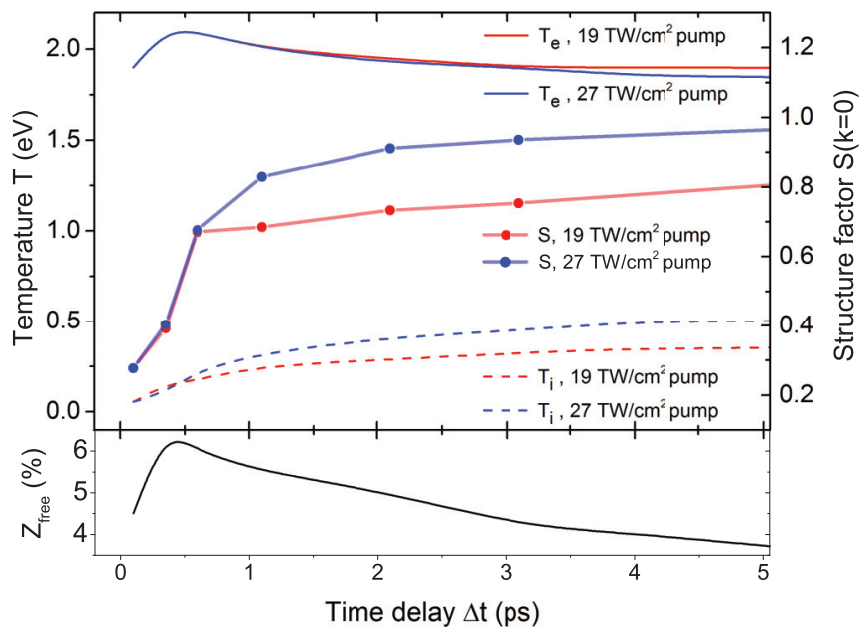

FIG. 3. (Color online) Simulation of the temporal evolution of the plasma parameters and the structure factor. The temperatures of the electronic and atomic-ionic subsystems are calculated with the one-dimensional radiation-hydrodynamics code HELIOS using PROPACEOS 4.2 [21], which calculates the ionization via a Saha model. Here $T_{e}$ and $T_{i}$ are the electron and ion temperatures, respectively. Also shown is the structure factor at $k=0$ (the lines connecting the data points are a guide to the eye). No temporal evolution in the initial ion density is observed. The ionization ranges between $4 \%$ and $6 \%$, temporally following the electron temperature dependence.

Here $m_{e}$ is the electrons mass and $h$ and $k_{B}$ are Planck's and Boltzmann's constants, respectively. Since for hydrogen the atomic number is $Z=1, Z_{\text {free }}$ is both the mean number of free electrons per atom and the mean ionization degree. The result is plotted in Fig. 4, together with calculations using the program package COMPTRA [23] that takes into account higher interaction corrections based on efficient interpolation formulas.

In the two EOS models used for the radiationhydrodynamics simulations, the most striking difference is ionization at low temperatures. Figure 4 shows the mean ionization $Z_{\text {free }}$ of hydrogen at $0.08 \mathrm{~g} / \mathrm{cm}^{3}$ for temperatures up

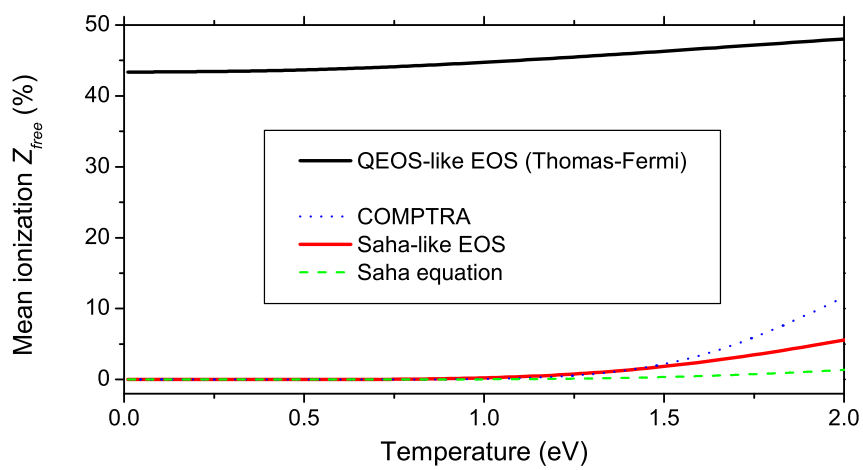

FIG. 4. (Color online) Mean ionization $Z_{\text {free }}$ as a function of plasma temperature $T$, comparing Saha-like and QEOS-like models for the EOS, as well as the predictions made by the Saha equation itself and the code COMPTRA [23]. QEOS predicts significant intrinsic pressure ionization, while all other models agree on less than $10 \%$ ionization for $T<2 \mathrm{eV}$. 
to $2 \mathrm{eV}$. The Saha-like EOS predicts no ionization at $T=0 \mathrm{eV}$ and values less than $10 \%$ at $T<2 \mathrm{eV}$. For ion temperatures of $T_{i}<1 \mathrm{eV}$ relevant in this work, the ionization of the Saha-like EOS is in good agreement with the results from both the analytic solution of Eq. (1) and COMPTRA.

In sharp contrast, the Thomas-Fermi model predicts initially $\sim 50 \%$ ionization at $T=0 \mathrm{eV}$. For higher densities the initial ionization further increases (not shown here). Hence QEOS yields pressure ionization for cryogenic hydrogen, which drastically alters energy partitioning between ions and electrons and increases the electron-ion energy transfer rate. However, condensed molecular hydrogen is a wide-band-gap insulator $\left(E_{g}=15 \mathrm{eV}\right)$ at ambient pressure and metallic properties are only observed at higher pressures exceeding $140 \mathrm{GPa}$ [24]. The Thomas-Fermi model assumes the energy or particle density to be constant over the Fermi screening length. This is only fulfilled for densities exceeding those relevant for our experiment. We conclude that the Thomas-Fermi model is not applicable for the small temperatures, low atomic numbers, and moderate densities as in our case. Finally, in the case of a sharply defined energy level (13.6 eV in our case), the Saha equation is superior to Thomas-Fermi model, which assumes a somewhat wide energy distribution for the binding energy, lowering the ionization threshold. In order to best fit the scattering data (see Sec. VIA) the simulations have been performed using the Saha-like EOS database PROPACEOS 4.2 [21], which predicts weak ionization between $Z_{\text {free }}=$ $4 \%$ and $6 \%$ (temporally following the electron temperature dependence as shown in Fig. 3) and consequently a weak electron-ion energy exchange (of the order of picoseconds) for the experiment considered in this paper.

\section{X-RAY PROBING}

As illustrated in Fig. 1, the probe pulse is focused onto the hydrogen jet at a defined time after the pump pulse has heated the target. At 92-eV photon energy, photoabsorption has the highest cross section with a small fraction of the incident radiation being also scattered. We use the scattered light of a delayed second pulse to probe the system.

\section{A. Experimental scattering spectra}

Scattering is collected at $90^{\circ}$ relative to the incident FEL radiation in the vertical plane to account for the horizontal polarization of the FEL [25]. To discriminate between the scattered XUV photons and plasma self-emission or energetic particles, we employ the XUV spectrometer HiTRaX [26]. It features a toroidal mirror $255 \mathrm{~mm}$ from the target, providing a large solid angle of $1.9 \times 10^{-3} \mathrm{sr}$. The spectral dispersion was calibrated in situ using plasma emission lines [27]. In Table II a summary of the optical elements and their efficiencies is provided. X-ray-scattering spectra are measured for various time delays up to 5 ps. Figure 5 shows a significant increase of the total scattered intensity between 0 and 1 ps time delay. We also notice that the spectral shape shows subtle changes of the wings, mostly dominated by the incident FEL spectrum.
TABLE II. Components of the HiTRaX spectrometer and their derived efficiency for a 13.5-nm wavelength.

\begin{tabular}{|c|c|}
\hline \multicolumn{2}{|c|}{ Nondispersive direction } \\
\hline Angular range & $4.49 \mathrm{deg}$ \\
\hline Collection angle & $78.4 \times 10^{-3} \mathrm{rad}$ \\
\hline \multicolumn{2}{|c|}{ Dispersive direction } \\
\hline Distance source mirror & $255 \mathrm{~mm}$ \\
\hline Mirror length & $50 \mathrm{~mm}$ \\
\hline Grazing angle & $7.3 \mathrm{deg}$ \\
\hline Effective mirror aperture & $6.35 \mathrm{~mm}$ \\
\hline Collection angle & $24.9 \times 10^{-3} \mathrm{rad}$ \\
\hline Total collection solid angle & $1.95 \times 10^{-3} \mathrm{sr}$ \\
\hline \multicolumn{2}{|c|}{ Transmissions } \\
\hline Toroidal mirror reflectivity & 0.78 \\
\hline Grating diffraction efficiency & 0.25 \\
\hline No filter & \\
\hline Total collection efficiency & $18.9 \times 10^{-3} \mathrm{sr}$ \\
\hline \multicolumn{2}{|c|}{ CCD camera } \\
\hline Photon energy & $92 \mathrm{eV}$ \\
\hline Band gap & $2.6 \mathrm{eV} /$ electron-hole pair \\
\hline AD conversion & 7 electron-hole pair/count \\
\hline Quantum efficiency & 0.42 \\
\hline Counts per photon & $1.5 \overline{3}$ \\
\hline Total counts per photon $\eta$ & $5.9 \times 10^{-4} \mathrm{sr}$ \\
\hline
\end{tabular}

\section{B. Thomson scattering}

The classical differential light scattering cross section $d \sigma$ per solid angle increment $d \Omega$ of a single electron is given as

$$
\frac{d \sigma}{d \Omega}=r_{0}^{2}\left(\boldsymbol{\epsilon}_{1} \cdot \boldsymbol{\epsilon}_{2}\right)^{2}
$$

where $r_{0}=2.82 \times 10^{-5} \AA$ is the classical electron radius and $\boldsymbol{\epsilon}_{1}$ and $\boldsymbol{\epsilon}_{2}$ are the polarization vectors of the incoming and outgoing waves. In our case, the incoming and outgoing $\mathrm{x}$ rays are linearly polarized in the horizontal direction, so their polarization vectors are parallel to each other $\left(\boldsymbol{\epsilon}_{1} \cdot \boldsymbol{\epsilon}_{2}=1\right)$. Hence the number of scattered photons $N_{\text {scat }}$ per solid angle

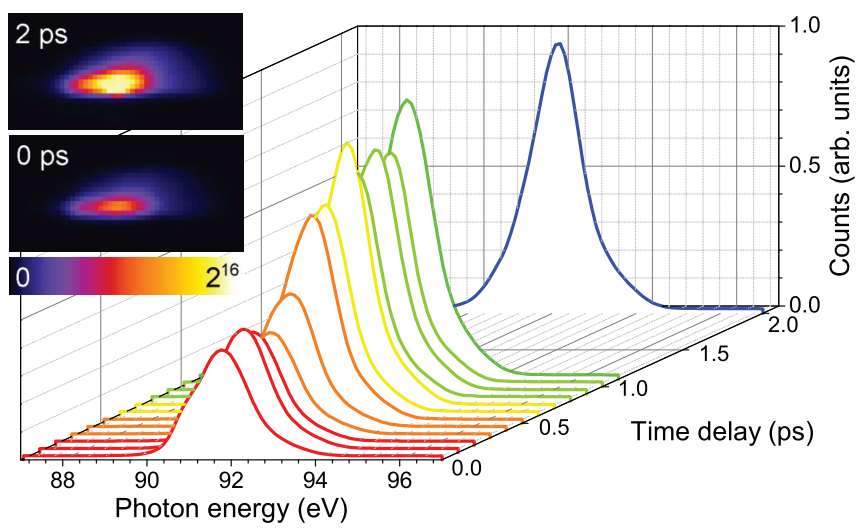

FIG. 5. (Color online) Experimental scattering spectra as a function of pump-probe delay, from Ref. [7]. The inset shows two 16-bit raw spectra, $25 \times 50$ pixels of $13.5 \mu \mathrm{m}^{2}$ in size, with a horizontally oriented spectral axis corresponding to the graph, where the spectral profiles are integrated along the vertical axis. 
$d \Omega$ and per incident photons $N_{\text {inc }}$ is given by [28]

$$
\frac{1}{N_{\text {inc }}} \frac{d N_{\text {scat }}(t)}{\mathrm{d} \Omega}=\frac{V n_{i}}{A} Z r_{0}^{2} \int d \omega S(k, \omega ; t),
$$

where $V$ is the scattering volume, $n_{i}$ is the heavy-particle density (ions and atoms), $A$ is the irradiated area (the focal spot being larger than the sample), $Z=1$ is the atomic number, $r_{0}$ is the classical electron radius, and $S(k, \omega)$ is the dynamic structure factor for a momentum transfer $k$, where $\omega$ denotes the frequency shift in the case of inelastic scattering.

\section{Structure factor}

From Eq. (3) it is evident that the temporal evolution of the measured $x$-ray scattering signal from the probe pulse is completely characterized by the time-dependent (dynamic) structure factor $S(k, \omega ; t)$. Following Chihara's approach [29,30], $S(k, \omega)$ can be expressed by a sum of independent contributions

$$
\begin{aligned}
S(k, \omega)= & \overbrace{Z_{\text {free }} S_{e e}^{0}(k, \omega)}^{\text {free electrons }}+\overbrace{|f(k)+q(k)|^{2} S_{i i}(k, \omega)}^{\text {bound electrons }} \\
& \underbrace{+Z_{\text {bound }} \int d \omega^{\prime} S_{c}(k, \omega) S_{s}\left(k, \omega-\omega^{\prime}\right)}_{\text {bound-free transitions(Raman) }} .
\end{aligned}
$$

In the first term, $S_{e e}^{0}(k, \omega)$ describes the scattering from free electrons weighted by $Z_{\text {free }}$, the mean number of free electrons per hydrogen atom. Due to the small ionized fraction of $Z_{\text {free }}<7 \%$ the scattering from free electrons may be neglected.

In the second term, $S_{i i}(k, \omega)$ characterizes the scattering from tightly bound electrons, weighted by the atomic form factor $f(k)$, and weakly bound electrons, weighted by the screening function $q(k)$. In the present case of a hydrogen plasma, we may assume [31]

$$
\lim _{k \rightarrow 0}|f(k)+q(k)|=Z_{\text {bound }}+Z_{\text {free }}=Z=1 .
$$

Hence $|f+q|^{2}=1$ is constant for $k \rightarrow 0$ and the signal is only dependent on $S_{i i}$.

The third term describes Raman-type transitions, where inner-shell electrons are excited to the continuum [31,32] (taken into account via the factor $S_{c}$ ), convoluted with the ion motion $S_{s}$. The Raman term is weighted by the mean number of bound electrons per atom $Z_{\text {bound }}$. For $92-\mathrm{eV}$ soft $\mathrm{x}$ rays, the maximum Compton recoil energy transfer of $\hbar \omega=$ $(\hbar k)^{2} / 2 m_{e}=33 \mathrm{meV}$ (in a backscatter event) is insufficient to excite bound electrons into the continuum.

Chihara's approach (4) is in general only valid for liquid metals, but we may use it for the ionic subsystem to describe the Thomson scattering from bound electrons. To define the $S^{\mathrm{mol}}(k, \omega)$ for the entire molecular system, the structure factor of the atoms $S_{\mathrm{HH}}$, the molecules $S_{\mathrm{H}_{2} \mathrm{H}_{2}}$, ions $S_{i i}$, and their different combinations $S_{i \mathrm{H}}, S_{i \mathrm{H}_{2}}, S_{\mathrm{H}_{2}}, S_{e i}, S_{e \mathrm{H}}$, and $S_{e \mathrm{H}_{2}}$ would be needed [33]. A term can be added to the Chihara formula to account for correlations between bound electrons:

$$
\begin{aligned}
S^{\mathrm{mol}}(k, \omega)= & \overbrace{Z_{\text {free }} S_{e e}^{0}(k, \omega)}^{\text {free electrons [see Eq. (4)] }}+\overbrace{Z_{\text {bound }} \int d \omega^{\prime} S_{c}(k, \omega) S_{s}\left(k, \omega-\omega^{\prime}\right)}^{\text {bound-free transitions [see Eq. (4)] }} \\
& \underbrace{2 \sum_{a} \sqrt{Z_{\text {free }} x_{a}} f_{a} S_{e a}(k, \omega)}_{\text {weakly bound electrons }}+\underbrace{\sum_{a, b} \sqrt{x_{a} x_{b}} f_{a} f_{b} S_{a b}(k, \omega)}_{\text {tightly bound electrons }},
\end{aligned}
$$

where we introduced the concentrations $x_{a}=n_{a} / \sum_{a} n_{a}$ for molecules with indices $a, b$. Here $f_{a}$ is the atomic or ionic form factor of bound states of component $a$. Ideally we would have to extract the corresponding structure factors from densityfunctional-theory (DFT) simulations (see Sec. V A), which has to be postponed to future work. As an approximation we therefore use only the structure factor of all heavy particles (the ion-ion structure factor as provided by the DFT simulations) without attempting to separate out different molecular species.

At a $90^{\circ}$ scattering angle, the momentum transfer amounts to $k=\left\|\mathbf{k}_{1}-\mathbf{k}_{2}\right\|=0.0348 a_{B}^{-1}$. Here $\mathbf{k}_{1}$ and $\mathbf{k}_{2}$ are the incident and scattered $\mathrm{x}$-ray wave numbers, respectively. For our conditions the probed length $\lambda^{*}=2 \pi / k=96 \AA$ is about 100 times larger than the mean interparticle distance $r$ on the order of angstroms. Therefore, we probe the collective behavior of a large number of particles. Since the value of $k$ is sufficiently close to zero we proceed with the structure factor at zero momentum transfer $S(k=0)$. The benefit is that, in contrast to $S(k>0), S(0)$ is a measure for the sum of all short- and long-range electron correlations and directly relates to the compressibility (see Sec. VD). The validity of this approach has been verified by classical hypernetted-chain (CHNC) calculations [34,35], which indicates no changes of the structure factor $S$ between $k=0.0348 a_{B}^{-1}$ and $k=0$. On the other hand, the probed length is about 2000 times smaller than the hydrogen jet diameter $D \sim 18 \mu \mathrm{m}$, so we are far from the classical Mie scattering regime, which would require $k D \sim 1$.

\section{CALCULATING STRUCTURAL PROPERTIES}

Radiation-hydrodynamics simulations provide the thermodynamic, macroscopic properties of a statistical system; however, to calculate the structure factor, which dictates the experimentally observed scattering via Eq. (3), knowledge of the microscopic structure of the hydrogen plasma is essential. 


\section{A. Density-functional theory}

The simulated particle density and the temperatures of electrons and ions or atoms from HELIOS are used as input parameters for $a b$ initio two-temperature (2T) DFT molecular dynamics (MD) simulations [36]. The code uses the physical picture, which considers only electrons and nuclei and all other features (e.g., bound states such as atoms and molecules) result from correlations. The use of two-temperature DFT is an alternative method [37] taking into account all equilibrium correlations, except the modification of wave functions imposed by nonequilibrium dynamics and relaxation. It treats all electrons for given arbitrary positions of the nuclei $r$. From the resulting electric fields $E(r)$ the Coulomb forces on the nuclei are derived and move these in the MD step.

For the 2T DFT MD we use the Vienna ab initio simulation package (VASP) [38]. Density-functional theory at finite temperatures is implemented in VASP solving the Kohn-Sham equations self-consistently to minimize the free energy $F[n(\mathbf{r})]$ of the system as a functional of the local electron density

$$
n(\mathbf{r})=\sum_{i=1}^{N} f\left(\epsilon_{i}, T\right)\left|\phi_{i}(\mathbf{r})\right|^{2},
$$

where the wave functions $\phi_{i}(\mathbf{r})$ are weighted with the Fermi distribution $f\left(\epsilon_{i}, T\right)$. The effective potential defining all interactions in the system includes ion-background contributions $v_{\text {ext }}(\mathbf{r})$, the Hartree energy, and the exchange-correlation functional $F_{\mathrm{XC}}[n(\mathbf{r})]$. The heavy-particle temperature $T_{i}$ is controlled with a Nosé thermostat [39], while the electron temperature $T_{e}$ is defined via the Fermi weighting of the electron distribution. Convergence is checked with respect to the particle number, the k-point sets used for the evaluation of the Brillouin zone, and the energy cutoff for the plane-wave basis set. The Coulomb interactions between the electrons and ions are treated using projector-augmented wave potentials [40] with a converged energy cutoff of $1400 \mathrm{eV}$. We chose 64 atoms and the Baldereschi mean value point [41] and use the exchange-correlation functional of Perdew, Burke, and Ernzerhof (PBE) [42], which has been shown to give reasonable results for warm dense matter states $[36,43]$. The ionization degree from HELIOS (as shown in Fig. 3) is not used as an input for DFT, but is derived independently from DFT.

\section{B. Pair distribution function $g(r)$}

Density-functional theory provides the radial pair distribution function $g(r)$ of atoms and ions. Figure 6 shows $g(r)$ for different temperatures, corresponding to different time delays. A pronounced peak in the distribution function is seen at radius $r \sim 0.75 \AA$, which coincides with the molecular bond [36] and characterizes an undissociated system. With increasing temperature the molecular peak drops and broadens, while a continuous radial distribution arises, corresponding to bond breakage and plasma formation. Figure 7 illustrates the respective positions of hydrogen atoms, according to $g(r)$. Hence, an ideal plasma state is reached if the pair distribution function is unity, e.g., all particles are randomly distributed.

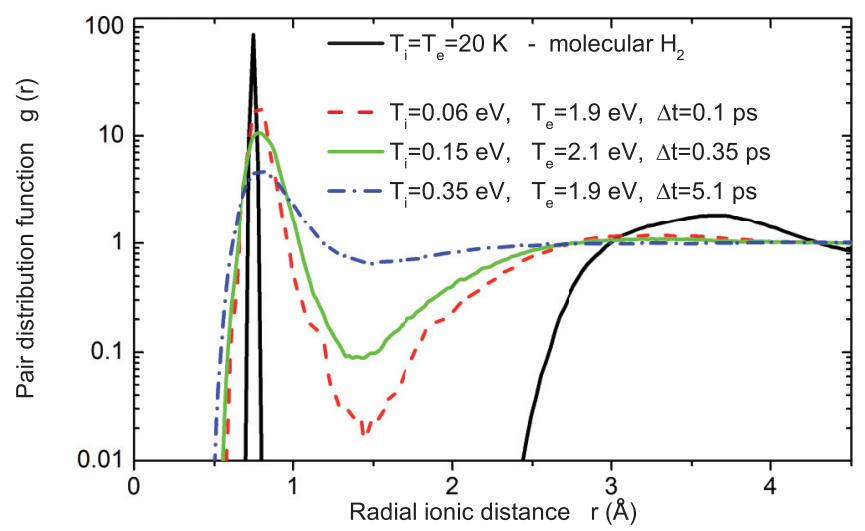

FIG. 6. (Color online) Calculated pair distribution function, from Ref. [7]. Shown is the pair distribution function $g(r)$ as a function of the radial interparticle distance $r$ for different time delays and temperatures. For cryogenic hydrogen $(T=20 \mathrm{~K})$, a peak at $r \sim$ $0.75 \AA$ indicates the distance in the $\mathrm{H}_{2}$ molecule. With increasing temperature the molecular bonds break and the intensity of the peak drops.

\section{Dissociation of molecules}

The hydrogen molecule has a strong covalent bond with a binding energy of $E_{\mathrm{H}-\mathrm{H}}=4.52 \mathrm{eV}$. In the DFT simulations, this binding energy is accounted for by solving Schrödinger's equation. If no FEL is applied, the system stays at cryogenic temperatures and therefore remains completely molecular (left panel in Fig. 7). When the FEL is applied, the bonds break due to the elevated electron and ion temperatures. These temperatures reflect a certain energy distribution, approaching Maxwell-Boltzmann statistics in local thermal equilibrium. A fraction of particles carries significant kinetic energy even at moderate temperatures. In an electronic system with $T_{e}=2 \mathrm{eV}$ a considerable number of electrons have energies exceeding the molecular bond energy $E_{\mathrm{H}-\mathrm{H}}$. This also applies for the ionic subsystem with temperatures $T_{i} \leqslant 1 \mathrm{eV}$. Figure 8 shows the dissociation degree for 2T DFT $\left(T_{e} \neq T_{i}\right)$ and 1T DFT ( $T_{e} \equiv T_{i}$ ) as function of delay time. In the two-temperature simulations the dissociation degree is higher due to the initially hotter electron system, which efficiently dissociates molecules via impact ionization and molecular vibrations. Evidently the temporal evolution of the correlations is strongly dependent on the energy partition between electrons and protons, requiring a two-temperature approach.

\section{Deriving $S(k)$ from $g(r)$}

In general, the structure factor $S(k)$ is related to the pair distributions $g(r)$ by [44]

$$
S(k)=\underbrace{1}_{\text {ideal system }}+\underbrace{n_{i} \int[g(r)-1] e^{\mathrm{i} \vec{k} \cdot \vec{r}} d \vec{r}}_{\text {correlations }} .
$$

Here $n_{i}$ is the density of all heavy particles (atoms and ions or protons). Equation ( 7 ) is composed of a constant contribution of an ideal system $(S \equiv 1)$ and the correlations, given by a Fourier transform of the total correlation function $g(r)-1$. 


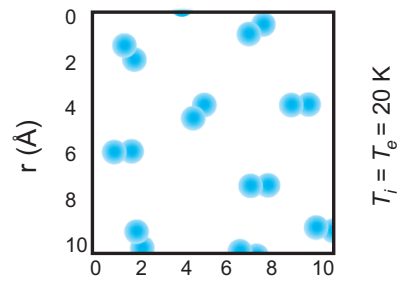

$r(\AA)$

cryogenic molecules

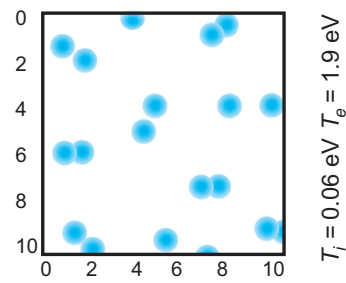

\begin{tabular}{|l}
0 ps \\
time delay (ps)
\end{tabular}
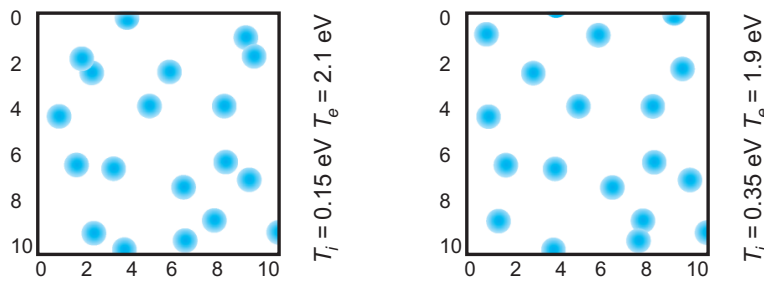

5 ps

FIG. 7. (Color online) Two-dimensional illustration of the hydrogen atoms. Shown is the transition from initial cryogenic hydrogen (left) to an atomic ideal plasma (right).

For zero momentum transfer $k=0$, this Fourier transform switches to an integration over the total correlation functions

$$
S(0)=1+n_{i} \int d \vec{r}[g(r)-1] .
$$

In Fig. 9 the structure factor $S(k)$ for different time delays extracted from the DFT simulations are shown. We see that for larger momentum transfer (e.g., higher photon energy and/or backscatter geometry) the structure factor gets less sensitive to the particle correlations. Highest sensitivity is achieved for $k=0$, as realized in this work.

For $k<1.25 / \AA$ the DFT simulations yield no consistent results for $S(k)$ due to the limited size of the simulation box, but for $k=0$ they are valid since $S(0)$ reduces to the ratio between the real $\kappa_{T}$ and the ideal $\kappa_{T}^{\text {id }}$ isothermal compressibilities

$$
S(0)=\frac{\kappa_{T}}{\kappa_{T}^{\mathrm{id}}} .
$$

The ideal compressibility is simply given by $\kappa_{T}^{\mathrm{id}}=1 / n_{i} k_{B} T$, while the real compressibility is obtained from the equation of state via $\kappa_{T}=(\partial \rho / \partial p) / \rho$ derived from the 2T DFT simulations as well ( $\rho$ is the mass density and $p$ the pressure). This means that $S(0)$ is related to the density change as a function of pressure. The compressibility can only be related to the total correlations for $r \rightarrow \infty$.

The predicted time dependence of $S(0)$ is shown in Fig. 3. The DFT signal follows the rise of the ion temperature; therefore, it is sensitive to the energy transfer from electrons to ions and atoms. At initial times, we see that $S(0)$ differs from

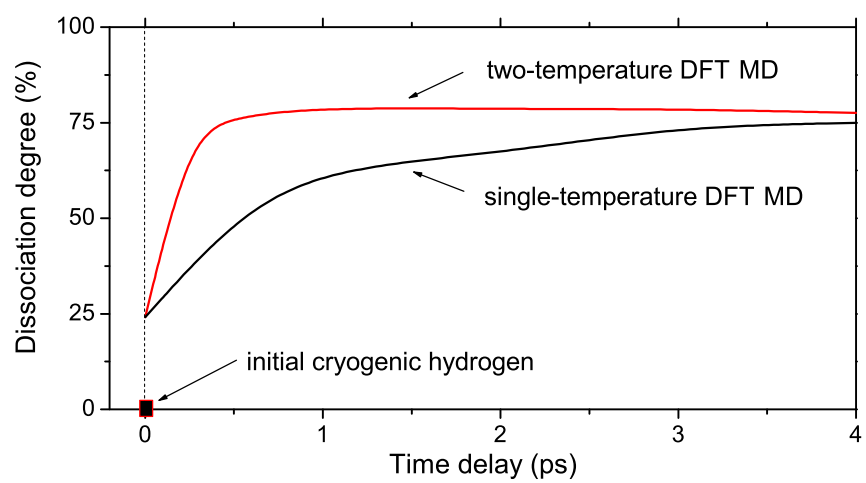

FIG. 8. (Color online) Dissociation degree of hydrogen molecules as a function of delay for 2T DFT $\left(T_{e} \neq T_{i}\right)$ and 1T DFT $\left(T_{e} \equiv T_{i}\right)$. unity, indicating a highly correlated system. At later times, $S(0)$ approaches 1 , which can be interpreted as a transition from molecular liquid hydrogen to an almost uncorrelated fluidlike structure [cf. Eq. (8)]. When comparing the $S(0)$ inferred from DFT to the ion temperature shown in Fig. 3, $S$ does not change very much once $T_{i}$ exceeds $0.25 \mathrm{eV}$ since beyond this point most of the molecular bonds are already broken (see also Fig. 8).

\section{E. Alternative approaches to calculate $S(0)$}

Figure 10 shows a comparison of the results for $S(0)$ calculated with 2T DFT, with the CHNC [34,35], and in the Salpeter approximation [45]. Note the logarithmic ordinate. As compared to 2T DFT MD, the Salpeter approximation predicts a structure factor that is too small and deformed, but the results of the $\mathrm{CHNC}$ are qualitatively similar to DFT. Since CHNC simulations take considerably less computational time as compared to DFT, they are suitable for fast predictions or for the future treatment of inhomogeneous targets.

\section{RESULTS}

\section{A. Experimental data}

In Fig. 11 the scattered fraction of photons is plotted versus time delay. The experimental data points were normalized to the incident photon number $N_{\text {inc }}$ (see Table I) and the XUV spectrometer efficiency $\eta$ (see Table II), calculated from the

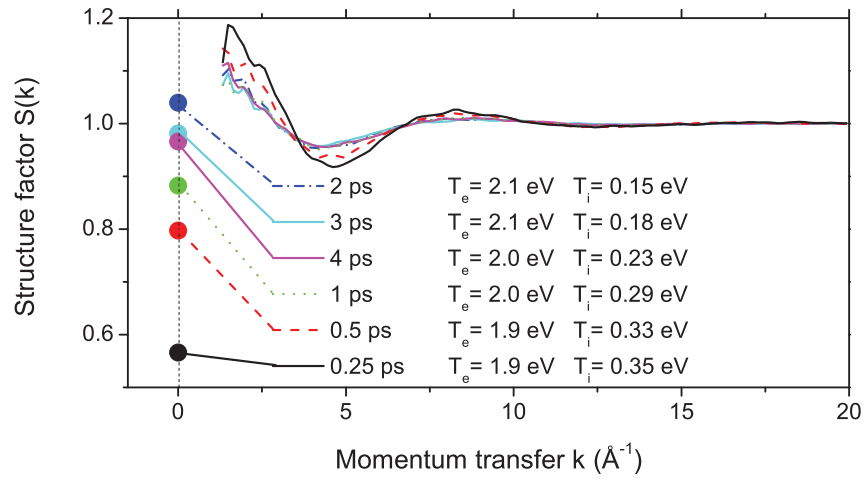

FIG. 9. (Color online) Calculated structure factor $S(k)$ for different time delays, extracted from the DFT simulations. For $k<1.25 / \AA$ the DFT simulations are not trustable, but for $k=0$ (points) they are valid. 


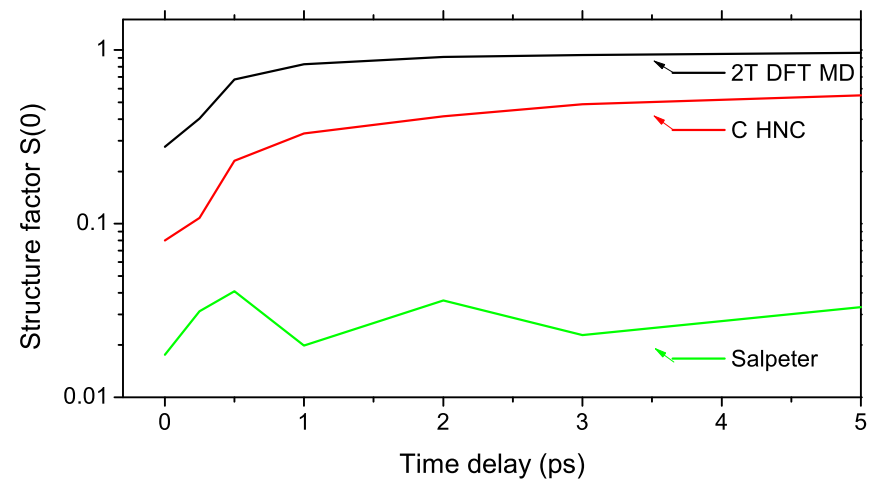

FIG. 10. (Color online) Structure factor $S(k=0)$ calculated with 2T DFT MD, with the CHNC, and in the Salpeter approximation.

specifications. The lines in Fig. 11 represent the simulated scattered fraction via Eq. (3). In this equation, hydrodynamics simulations yield the time-dependent plasma parameters that enter into DFT MD to derive $S(0)$ for each time step (sec. V D).

We observe a significant increase of the signal for times $\leqslant 1$ ps. Stronger pumping of the liquid hydrogen (corresponding to $27 \mathrm{TW} / \mathrm{cm}^{2}$ ) leads to more scatter because the particles are less ordered. For weaker pumping $\left(19 \mathrm{TW} / \mathrm{cm}^{2}\right)$, we were able to measure time delays up to 5 ps. First we observe that after $1 \mathrm{ps}$ the scattering amplitude stops growing, flattens until about 2 ps, and slowly decreases at 5 ps. This decrease could be attributed to recombination processes in weakly ionized

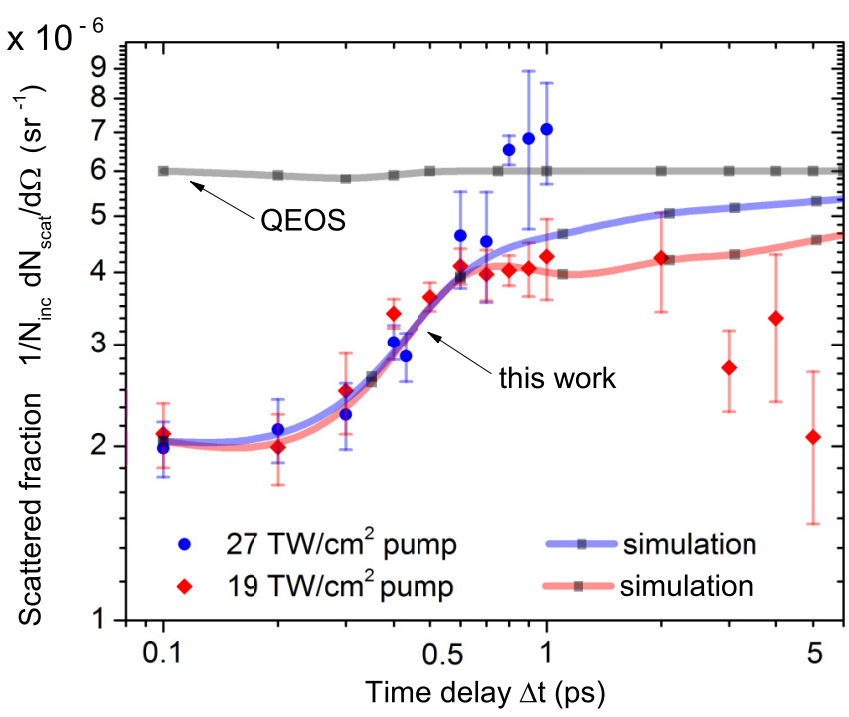

FIG. 11. (Color online) Temporal evolution of the measured (points) and simulated (lines) x-ray scattering, from Ref. [7]. A total of 300 exposures were grouped together by delay and their errors resemble their root mean square deviations. For the blue diamonds, ranging from $\Delta t=0$ to $1 \mathrm{ps}$ only, the more intense $27-\mathrm{TW} / \mathrm{cm}^{2}$ pulse pumps the target, while it is probed by the $19-\mathrm{TW} / \mathrm{cm}^{2}$ pulse. The red points, ranging from 0 to $5 \mathrm{ps}$, resemble the inverse case where the pump was less intense $\left(19 \mathrm{TW} / \mathrm{cm}^{2}\right)$ than the probe $\left(27 \mathrm{TW} / \mathrm{cm}^{2}\right)$. In both cases the signal increases within $1 \mathrm{ps}$ and for the more intense pump the amplitude is larger. The lines represent the simulated scattered fraction via Eq. (3), the red and blue (lower) ones using a Saha-like ionization model and the gray (upper) one using QEOS. hydrogen not currently implemented in our hydrodynamics calculations. Best agreement with the simulations (as shown in Fig. 11) is achieved when multiplying the measured values by a factor of 2.6. We attribute this mainly to fluctuations of the hydrogen jet (FEL pulses miss the jet center) and the FEL radiation (some FEL pulses have low energy or poor pointing stability).

A Gaussian fit to the experimental data yields a valley-topeak time of $1.1 \pm 0.2 \mathrm{ps}$. After deconvolution of the probe pulse duration, we derive that cryogenic molecular hydrogen responds to an impulsive heating of the electrons within $0.9 \pm$ $0.2 \mathrm{ps}$.

\section{B. Equilibration time and ac conductivity}

The hydrodynamics simulations employ a Spitzer-like equilibration time, slightly modified by the heat capacity [21]. The classical Spitzer expression describing a fully ionized plasma is

$$
\tau=\frac{32 \sqrt{2} \pi \epsilon_{0}^{2} m_{i}}{\sqrt{m_{e}} e^{4}} \frac{1}{Z_{\text {free }}^{2} n_{i} \ln \Lambda}\left(k_{B} T_{e}\right)^{3 / 2},
$$

with $\ln \Lambda$ being the Coulomb logarithm. Using the classic expression, the latter becomes negative for degenerate plasmas, e.g., at low temperatures and high densities, which is unphysical. To overcome this limitation, improved expressions for $\ln \Lambda$ have been proposed [46,47], yielding $1 \leqslant \ln \Lambda \leqslant 8$ for our conditions. For a partially ionized hydrogen plasma, the fully ionized subplasma is considered by $n_{i}=Z_{\text {free }} n_{h}$. Figure 11 shows that the simulations using the Saha-like EOS and the Spitzer model (red and blue lines) are in good agreement with the measured temporal evolution of the scattering amplitude.

We further prove that a Saha ionization model as implemented in this work is superior to the Thomas-Fermi model as implemented in the QEOS [22]. The latter predicts about order of magnitude too short equilibration times, generating a plasma with $S(0) \sim 1$ well within the FEL pulse duration. Hence no pump-probe delay dependence for $S(0)$ is predicted using the QEOS (see Fig. 11).

It is not self-evident that the equilibration time derived from hydrodynamics simulations agrees with the DFT MD results because the Spitzer model (10) is not explicitly implemented in the latter. Since the DFT-simulated conductivity of hydrogen system shows a Drude-like behavior (not shown), the mean electron-ion collision time can be derived from a Drude fit at fixed time delay [48].

The ac conductivity $\sigma(\omega)$ for frequency $\omega$ is described by the Drude model by [49]

$$
\sigma(\omega)=\frac{\sigma(\omega=0)}{1+\left(\omega \tau_{\mathrm{c}}\right)^{2}},
$$

where $\tau_{\mathrm{c}}$ is the collision time. With the static (dc) electrical conductivity from the DFT data at $\omega=0$, also the free-electron density $n_{e}$ and thus $Z_{\text {free }}$ can be calculated via

$$
\sigma(\omega=0) \equiv \sigma_{0}=\frac{n_{e} e^{2} \tau_{c}}{m_{e}}
$$

where $m_{e}$ is the electron mass and $\tau_{c}$ is calculated from Eq. (11). As shown in Fig. 12, the mean ionization degree 


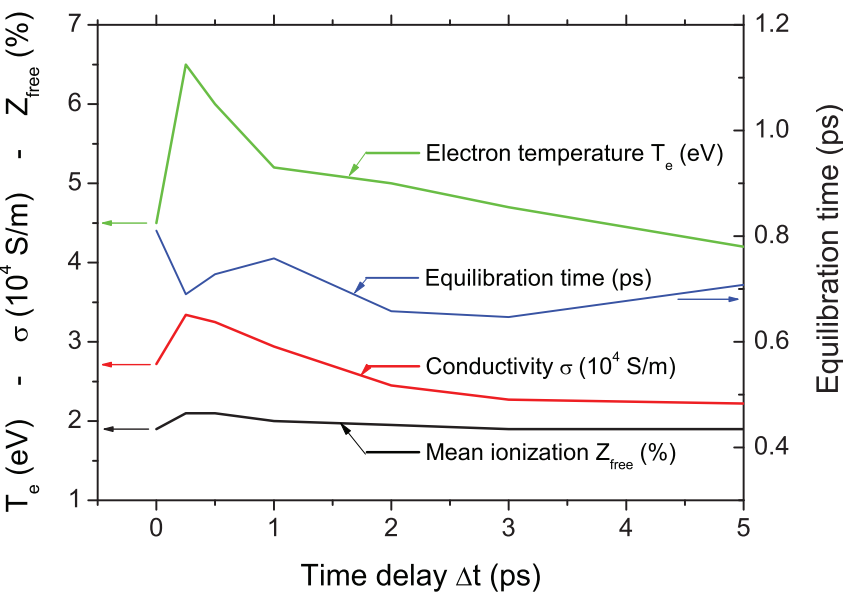

FIG. 12. (Color online) Temporal evolution of the plasma parameters derived from DFT MD. The electron temperature $T_{e}$ is an input parameter from HELIOS. The ionization degree $Z_{\text {free }}$ and the plasma conductivity $\sigma$ follow the temporal evolution of $T_{e}$ (left axis). On the right axis, the equilibration time is plotted, while the collision time is $10^{-3}$ times the equilibration time.

$Z_{\text {free }}$ and the plasma conductivity $\sigma$ (after PBE; see Sec. V A) follow the temporal evolution of $T_{e}$ (left axis). The ionization degree of $4.5 \%-6.5 \%$ [49] is consistent with ionization using the Saha-like EOS, yielding values between $4 \%$ and $6 \%$ (cf. Fig. 3). The equilibration times $\tau$ range between $0.65 \leqslant$ $\tau \leqslant 0.81$ ps (scaling as $1 / Z_{\text {free }}$ ), in good agreement with our measured value of $0.9 \pm 0.2$ ps. Heating of the atoms and ions takes of order $m_{p} / 2 m_{e} \sim 10^{3}$ longer than the time between individual electron-ion collisions because it takes many collisions, in particular if electrons collide with protons.

\section{CONCLUSION AND OUTLOOK}

We resolved the ultrafast phase transition from a molecular liquid to a dense atomic plasma in hydrogen with final ion temperature $T_{i} \leqslant 1 \mathrm{eV}$ at $0.08-\mathrm{g} / \mathrm{cm}^{3}$ solid density. These conditions do not catch the laboratory equivalent of ICF plasmas or Jovian planet interiors, but nevertheless allow inferring transport properties such as electron heating, equilibration dynamics, and plasma conductivity.

We showed that driving hydrogen from initially cryogenic condensed matter to an ideal plasma poses several challenges to the employed models. We evaluated ionization models for the equation of state data and showed that a Saha-like model is superior to a Thomas-Fermi approach as implemented in the QEOS. The QEOS predicts an initially metallic state with $\sim 40 \%$ ionization, in conflict with experimental data. In principle it accounts for degenerate plasmas, important in the framework of warm and dense matter, but is not accurate for the low temperatures and low atomic numbers found in astrophysically relevant plasmas, dealt with in this work.

In this regime, also the concept of the classical Coulomb logarithm $\ln \Lambda$ breaks down. It needs to be expressed by alternative approaches [50,51] as it enters the hydrodynamics simulations via the Spitzer conductivity model. We have shown that values of $1 \leqslant \Lambda \leqslant 8$ yield equilibration times $\tau$ consistent with both measurements and DFT MD results. Finally, an independent treatment of electron and ion temperatures in DFT MD was shown to be necessary for accurate $a b$ initio calculations of the microscopic properties such as dissociation and hence the structure factor.

Numerical simulations indicate that the Rayleigh-Taylor growth is highly sensitive to thermal conduction [52]. Reduction in the heat diffusion coefficient by a factor 10 corresponds to an increase by a factor $\sim 2$ in the mixing layer between compressed DT and beryllium in ICF capsules. In the same way, this is also expected to change the predicted depth of mixing layers in Jovian planets $[3,6]$. Hence, our results provide important insights and needed experimental data on transport effects, which will shed light on the detailed understanding of dense plasmas.

\section{ACKNOWLEDGMENTS}

The authors thank the FLASH machine and experiment team for their great support. The assistance of the Bundesministerium für Bildung und Forschung within the priority research area FSP 301 FLASH, the Deutsche Forschungsgemeinschaft within the SFB 652, CUI, and the VolkswagenStiftung is acknowledged. This work received partial funding from UK EPSRC through Grant No. EP/G007187/1 and from the French Agence Nationale de la Recherche under Grant No. IRONFEL - ANR-12-PDOC-0011. This work was partially performed under the auspices of the U.S. Department of Energy by Lawrence Livermore National Laboratory under Contract No. DE-AC52-07NA27344 and was supported by LDRD through Grant No. 11-ERD-050. It was partially supported by the US DOE Office of Science, Fusion Energy Sciences under Grant No. FWP 100182. Density-functional theory MD simulations were performed at the North-German Supercomputing Alliance (HLRN). Supporting simulations were performed at the John von Neumann-Institut for computing.
[1] N. Nettelmann et al., Astrophys. J. 683, 1217 (2008).

[2] J. D. Lindl et al., Phys. Plasmas 11, 339 (2004).

[3] J. Leconte and G. Chabrier, Astron. Astrophys. A 540, 20 (2012).

[4] A. J. Mackinnon, J. L. Kline et al., Phys. Rev. Lett. 108, 215005 (2012).

[5] S. H. Glenzer, D. A. Callahan, A. J. MacKinnon, J. L. Kline, G. Grim, E. T. Alger, R. L. Berger, L. A. Bernstein, R. Betti, D. L. Bleuel et al., Phys. Plasmas 19, 056318 (2012).
[6] S. P. Regan et al., Phys. Plasmas 19, 056307 (2012).

[7] U. Zastrau, P. Sperling, M. Harmand et al., Phys. Rev. Lett. 112, 105002 (2014)

[8] A. Kritcher et al., Science 322, 69 (2008).

[9] S. H. Glenzer et al., Phys. Rev. Lett. 98, 065002 (2007).

[10] G. Gregori et al., Phys. Rev. Lett. 101, 045003 (2008).

[11] E. García Saiz, G. Gregori, D. O. Gericke, J. Vorberger, B. Barbrel, R. J. Clarke, R. R. Freeman, S. H. Glenzer, F. Y. Khattak, M. Koenig et al., Nat. Phys. 4, 940 (2008). 
[12] B. Nagler, U. Zastrau, R. R. Fäustlin et al., Nat. Phys. 5, 693 (2009).

[13] S. M. Vinko, O. Ciricosta, B. I. Cho, K. Engelhorn, H. K. Chung, C. R. D. Brown, T. Burian, J. Chalupsk, R. W. Falcone, C. Graves et al., Nature (London) 482, 59 (2012).

[14] W. Ackermann et al., Nat. Photon. 1, 336 (2007).

[15] M. Wöstmann, R. Mitzner, T. Noll, S. Roling, B. Siemer, F. Siewert, S. Eppenhoff, F. Wahlert, and H. Zacharias, J. Phys. B 46, 164005 (2013).

[16] K. Tiedtke, A. Azima et al., New J. Phys. 11, 023029 (2009).

[17] R. Mitzner, A. A. Sorokin, B. Siemer, S. Roling, M. Rutkowski, H. Zacharias, M. Neeb, T. Noll, F. Siewert, W. Eberhardt, M. Richter, P. Juranic, K. Tiedtke, and J. Feldhaus, Phys. Rev. A 80, 025402 (2009).

[18] S. Toleikis, R. R. Fäustlin et al., High Energy Density Phys. 6, 15 (2010).

[19] P. Sperling et al., High Energy Density Phys. 7, 145 (2011).

[20] R. R. Fäustlin, Th. Bornath, T. Döppner, S. Düsterer, E. Förster, C. Fortmann, S. H. Glenzer, S. Göde, G. Gregori, R. Irsig et al., Phys. Rev. Lett. 104, 125002 (2010).

[21] J. J. MacFarlane, I. E. Golovkin, and P. R. Woodruff, J. Quant. Spectrosc. Radiat. Transfer 99, 381 (2006).

[22] R. M. More, K. H. Warren, D. A. Young, and G. B. Zimmerman, Phys. Fluids 31, 3059 (1988).

[23] S. Kuhlbrodt, B. Holst, and R. Redmer, Contrib. Plasma Phys. 45, 73 (2005).

[24] S. T. Weir, A. C. Mitchell, and W. J. Nellis, Phys. Rev. Lett. 76, 1860 (1996).

[25] A. Höll, Th. Bornath et al., High Energy Density Phys. 3, 120 (2007).

[26] R. R. Fäustlin, U. Zastrau, S. Toleikis, I. Uschmann, E. Förster, and Th. Tschentscher, J. Inst. 5, P02004 (2010).

[27] U. Zastrau et al., J. Inst. 6, P10001 (2011).

[28] S. H. Glenzer and R. Redmer, Rev. Mod. Phys. 81, 1625 (2009).

[29] J. Chihara, J. Phys. F 17, 295 (1987).

[30] J. Chihara, J. Phys.: Condens. Matter 12, 231 (2000).

[31] G. Gregori, S. H. Glenzer et al., Phys. Plasmas 11, 2754 (2004).

[32] S. Sahoo, G. F. Gribakin, G. Shabbir Naz, J. Kohanoff, and D. Riley, Phys. Rev. E 77, 046402 (2008).
[33] K. Wünsch, J. Vorberger, G. Gregori, and D. O. Gericke, Europhys. Lett. 94, 25001 (2011).

[34] M. W. C. Dharma-wardana and F. Perrot, Phys. Rev. Lett. 84, 959 (2000).

[35] M. W. C. Dharma-wardana and M. S. Murillo, Phys. Rev. E 77, 026401 (2008).

[36] B. Holst, R. Redmer, and M. P. Desjarlais, Phys. Rev. B 77, 184201 (2008).

[37] Z. Chen, B. Holst, S. E. Kirkwood, V. Sametoglu, M. Reid, Y. Y. Tsui, V. Recoules, and A. Ng, Phys. Rev. Lett. 110, 135001 (2013).

[38] G. Kresse and J. Furthmüller, Phys. Rev. B 54, 11169 (1996).

[39] S. Nosé, J. Chem. Phys. 81, 511 (1984).

[40] P. E. Blöchl, Phys. Rev. B 50, 17953 (1994).

[41] A. Baldereschi, Phys. Rev. B 7, 5212 (1973).

[42] J. P. Perdew, K. Burke, and M. Ernzerhof, Phys. Rev. Lett. 77, 3865 (1996).

[43] W. Lorenzen, B. Holst, and R. Redmer, Phys. Rev. B 82, 195107 (2010).

[44] M. Shimoji, Liquid Metals: An Introduction to the Physics and Chemistry of Metals in the Liquid State, Vol. 266 (Academic, London, 1977).

[45] D. E. Evans and J. Katzenstein, Rep. Prog. Phys. 32, 207 (1969).

[46] M. R. Zaghloul, M. A. Bourham, and J. M. Doster, Phys. Lett. A 268, 375 (2000).

[47] D. O. Gericke, M. S. Murillo, and M. Schlanges, Phys. Rev. E 65, 036418 (2002).

[48] K. U. Plagemann, P. Sperling, R. Thiele, M. P. Desjarlais, C. Fortmann, T. Döppner, H. J. Lee, S. H. Glenzer, and R. Redmer, New J. Phys. 14, 055020 (2012).

[49] P. Renaudin, V. Recoules, P. Noiret, and J. Clérouin, Phys. Rev. E 73, 056403 (2006).

[50] Y. T. Lee and R. M. More, Phys. Fluids 27, 1273 (1984).

[51] A. Esser, R. Redmer, and G. Röpke, Contrib. Plasma Phys. 43, 33 (2003).

[52] B. A. Hammel, S. W. Haan, D. S. Clark, M. J. Edwards, S. H. Langer, M. M. Marinak, M. V. Patel, J. D. Salmonson, and H. A. Scott, High Energy Density Phys. 6, 171 (2010). 